\title{
Quantum-Behaved Particle Swarm Optimization Algorithm with Controlled Diversity
}

\author{
Jun Sun, Wenbo Xu, and Wei Fang \\ Center of Intelligent and High Performance Computing, \\ School of Information Technology, Southern Yangtze University, \\ No. 1800, Lihudadao Road, Wuxi Jiangsu 214122, China \\ \{sunjun_wx, xwb_sytu, wxfangwei\}@hotmail.com
}

\begin{abstract}
Premature convergence, the major problem that confronts evolutionary algorithms, is also encountered with the Particle Swarm Optimization (PSO) algorithm. In the previous work [11], [12], [13], the Quantum-behaved Particle Swarm (QPSO) is proposed. This novel algorithm is a global-convergence-guaranteed and has a better search ability than the original PSO. But like other evolutionary optimization technique, premature in the QPSO is also inevitable. In this paper, we propose a method of controlling the diversity to enable particles to escape the sub-optima more easily. Before describing the new method, we first introduce the origin and development of the PSO and QPSO. The Diversity-Controlled QPSO, along with the PSO and QPSO is tested on several benchmark functions for performance comparison. The experiment results testify that the DCQPSO outperforms the PSO and QPSO.
\end{abstract}

\section{Introduction}

The Particle Swarm Optimization (PSO), first introduced by Kennedy and Eberhart [5], is a stochastic optimization that can be likened to the behavior of a flock of birds. It has been used to solve a range of optimization problems, including neural network training [6] and function minimization. Since its origin in 1995, several attempts have been made to improve the performance of the original PSO ([1], [2], [4], [8], [9], [10], [17]). The original PSO, however, is not a global optimization algorithm, as has been demonstrated by F. van den Bergh in [3]. In our previous work [11], [12], [13], we proposed a novel global-convergence-guaranteed PSO, Quantum-behaved Particle Swarm Optimization (QPSO).

Like other evolutionary algorithm, the PSO as well as QPSO, confront the problem of premature convergence, which results in great performance loss and sub-optimal solutions. In the PSO or QPSO, the fast information flow between particles seems to be the reason for clustering of particles. Diversity declines rapidly, leaving the PSO or QPSO algorithm leads to low diversity with fitness stagnation as an overall result. In this paper, we propose a Diversity-Controlled Quantum-behaved Particle Swarm Optimization (DCQPSO). In the DCQPSO, a threshold value was set for population's diversity measure to prevent premature convergence and therefore enhance the overall performance of the QPSO. The rest part of the paper is organized as follows. In the 
next section, the PSO is introduced. The origin and development of QPSO is described in Section 3 and the DCQPSOs are proposed in Section 4. Section 5 is the experiment results and discussion. Some conclusion remarks are given in Section 6.

\section{PSO Algorithms}

The PSO is a population-based optimization technique, where a population is called a swarm. Each particle represents a possible solution to the optimization task at hand. During each iteration each particle accelerates in the direction of its own personal best solution found so far, as well as in the direction of the global best position discovered so far by any of the particles in the swarm. Let $M$ denote the swarm size. Each individual $i(1 \leq i \leq M)$ has the following attributes: A current position in the search space $X_{i}=\left(x_{i, 1}, x_{i, 2}, \cdots, x_{i, n}\right)$, a current velocity $V_{i}=\left(v_{i, 1}, v_{i, 2}, \cdots, v_{i, n}\right)$, and a personal best position in the search space $P_{i}=\left(p_{i, 1}, p_{i, 2}, \cdots, p_{i, n}\right)$. During each iteration, each particle is swarm is updated using (1) and (2). Assuming that the function $f$ is to be minimized, that the swarm consists of $n$ particles, and that $r_{1} \sim U(0,1), r_{2} \sim U(0,1)$ are elements from two uniform random sequences in the range $(0,1)$, then

$$
v_{i, j}(t+1)=w \cdot v_{i, j}(t)+c_{1} \cdot r_{1, i}(t) \cdot\left[p_{i, j}(t)-x_{i, j}(t)\right]+c_{2} \cdot r_{2, i}(t) \cdot\left[p_{g, j}(t)-x_{i, j}(t)\right]
$$

for all $j \in 1,2 \cdots, n$, thus, $v_{i, j}$ is the velocity of the $j$ th component of the velocity of the $i$ th particle, and $c_{1}$ and $c_{2}$ denote the acceleration coefficients. $P_{g}$ is the global best position found by any particle during all previous steps. The new position of a particle is calculated using

$$
X_{i}(t+1)=X_{i}(t)+V_{i}(t+1)
$$

The value of each component in every $V_{i}$ vector can be clamped to the range $\left[-v_{\max }, v_{\max }\right]$ to reduce the likelihood of particles leaving the search space. The variable $w$ in (1) is called the inertia weight, whose value is typically setup to vary linearly from 1 to near 0 during the course of training run. Acceleration coefficients $c_{1}$ and $c_{2}$ also control how far a particle will move in a single iteration.

\section{Quantum-Behaved Particle Swarm Optimization}

Trajectory analyses in [5] demonstrated that, to guarantee convergence of the PSO algorithm, each particle must converge to its local attractor $q=\left(q_{1}, q_{2}, \cdots q_{D}\right)$, of which the coordinates are:

$$
q_{d}=\left(c_{1} r_{1} p_{i d}+c_{2} r_{2} p_{g d}\right) /\left(c_{1} r_{1}+c_{2} r_{2}\right),(d=1,2, \ldots \mathrm{n})
$$

or

$$
q_{d}=\varphi \cdot p_{i d}+(1-\varphi) \cdot p_{g d}, \varphi \sim U(0,1),(d=1,2, \ldots n)
$$


Assume that there is one-dimensional Delta potential well on each dimension at point $q$ and each particle has quantum behavior. For simplicity, we consider a particle in one-dimensional space, with point $q$ the center of potential well. Solving the Schrödinger equation, we can get the normalized the following probability distribution function $\mathrm{F}$

$$
F(y)=\int_{-\infty}^{y} Q(y) d y=e^{-2|q-x| / L}
$$

where $L$ determines search scope of each particle. Employing Monte Carlo method, we can obtain the position of the particle

$$
x=q \pm \frac{L}{2} \ln (1 / u) \quad u=\operatorname{rand}(0,1)
$$

where $u$ is a random number uniformly distributed in $(0,1)$.

A global point called Mainstream Thought or Mean Best Position of the population is introduced into PSO. The global point, denoted as mbest, is defined as the mean of the pbest positions of all particles. That is

$$
\text { mbest }=\frac{1}{M} \sum_{i=1}^{M} P_{i}=\left(\frac{1}{M} \sum_{i=1}^{M} p_{i 1}, \frac{1}{M} \sum_{i=1}^{M} p_{i 2}, \cdots, \frac{1}{M} \sum_{i=1}^{M} p_{i d}\right)
$$

where $M$ is the population size and $P_{i}$ is the pbest position of particle $i$. Then the value of $L$ and the position are evaluated by

$$
\begin{gathered}
L=2 \beta \cdot \mid \text { mbest }-x \mid \\
x:=q \pm \beta \cdot \mid \text { mbest }-x \mid \cdot \ln (1 / u)
\end{gathered}
$$

where parameter $\beta$ is called Contraction-Expansion Coefficient, which could be tuned to control the convergence speed of the algorithms. The PSO with equation (19) is called Quantum-behaved Particle Swarm Optimization (QPSO).

\section{Diversity-Controlled QPSO}

As we know, a major problem with PSO and other evolutionary algorithms in multimodal optimization is premature convergence, which results in great performance loss and sub-optimal solutions. In a PSO system, with the fast information flow between particles due to its collectiveness, diversity of the particle swarm declines rapidly, leaving the PSO algorithm with great difficulties of escaping local optima. Therefore, the collectiveness of particles leads to low diversity with fitness stagnation as an overall result. In QPSO, although the search space of an individual particle at each iteration is the whole feasible solution space of the problem, diversity loss of the whole population is also inevitable due to the collectiveness.

In 2002, Ursem has proposed a model called Diversity-Guided Evolutionary Algorithm (DGEA) [14], which applies diversity-decreasing operators (selection, 
recombination) and diversity-increasing operators (mutation) to alternate between two modes based on a distance-to-average-point measure. The performance of the DGEA clearly shows its potential in multi-modal optimization.

Also in 2002, Riget et al [15] adopted the idea from Usrem into the basic PSO model with the decreasing and increasing diversity operators used to control the population. This modified model of PSO uses a diversity measure to have the algorithm alternate between exploring and exploiting behavior. They introduced two phases: attraction and repulsion and the swarm alternate between these phases according to its diversity. The improved PSO algorithm is called Attraction and Repulsion PSO (ARPSO) algorithm.

Inspired by works undertaken by Ursem and Riget et al, we introduce the Diversity-Controlled model in Quantum-behaved PSO. The diversity is measure by the following formula.

$$
\text { diversity }(S)=\frac{1}{|S| \cdot|A|} \cdot \sum_{i=1}^{|S|} \sqrt{\sum_{j=1}^{D}\left(x_{i j}-\overline{x_{j}}\right)^{2}}
$$

where $S$ is the swarm, $|S|=M$ is the population size, $|A|$ is the length of longest the diagonal in the search space, $D$ is the dimensionality of the problem, $x_{i j}$ is the $j$ th value of the $i$ th particle and $\overline{x_{j}}$ is the $j$ th value of the average point.

But unlike to their works, we only set a low bound to the diversity of the population. The procedure of the algorithm is as follows. After initialization, the algorithm is running in attraction mode that guaranteed by setting the value of $\beta$ smaller than 1.0. In [13], using stochastic simulation, we found out that the particle will converge when $\beta<1.778$ and otherwise will diverge. In this paper, the attraction mode is realized by varying $\beta$ from 1.0 to 0.5 over the running. That is,

$$
\beta=(1.0-0.5) \times(\text { MAXITER }-t) / \text { MAXITER }-0.5
$$

where MAXITER is the maximum number of iterations and $t$ is the number of current iteration. In the course of the running of the algorithm, if the diversity measure of the population declines to below the threshold value dlow, the particles will explode to increase the diversity until it is larger than dlow. There are two method of making the particle to explode. One method is to control the ContractionExpansion Coefficient $\beta$. That is, we can set $\beta=\beta_{0}\left(\beta_{0}>1.778\right)$ once the diversity is lower than the threshold value $\mathrm{d}$ low. In this paper the DCQPSO using this method is called DCQPSO1. The other method of increasing the diversity is initializing the Mean Best Position (mbest) of the population across the search space once the diversity is smaller than $\mathrm{d}$ low. The reason for the initialization of mbest is that when the diversity is low, the distance between the particle and the mbest position is too small for the particle to escape the local optima as can be seen from equation (9). Therefore initializing the mbest could enlarge the gaps between particles and the mbest position, consequently making particles explode temporarily. The DCQPSO with this operation is called DCQPSO2. The DCQPSO algorithms are formulated as follows. 


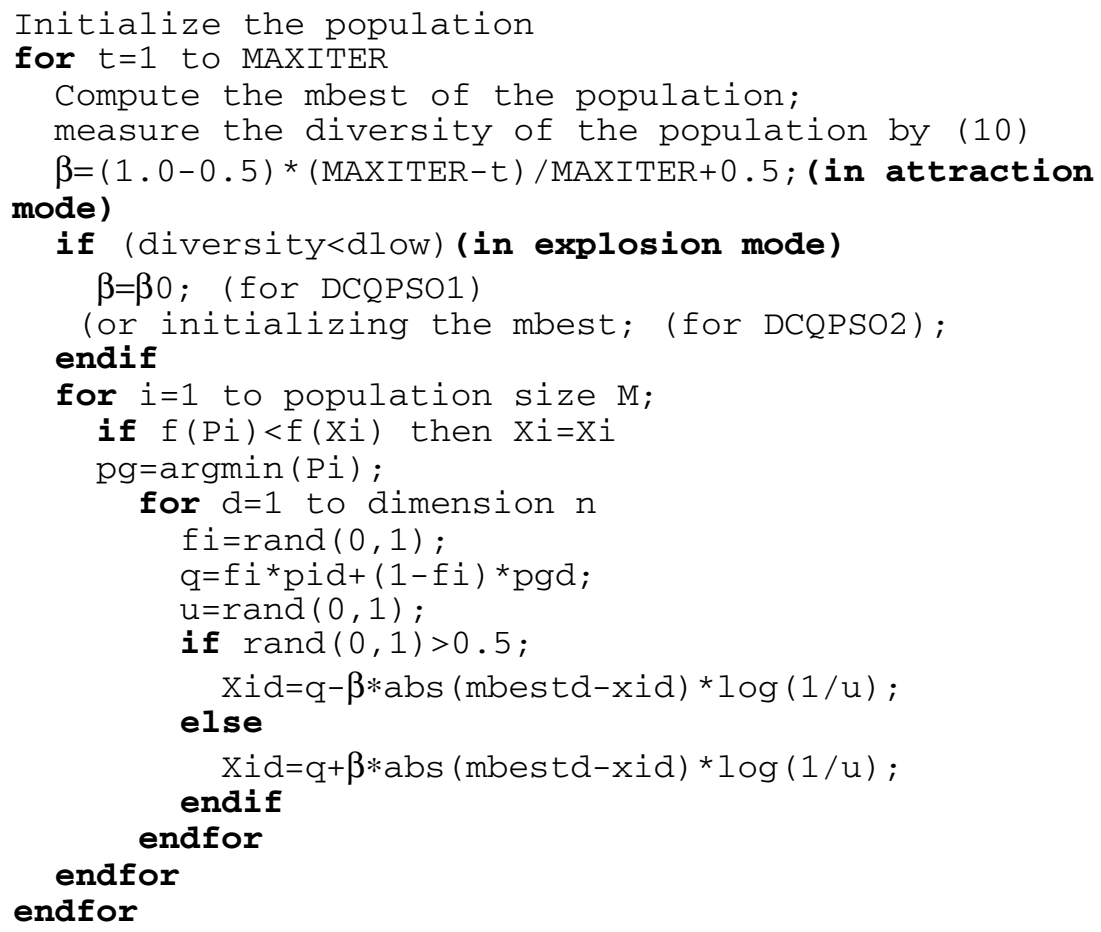

It can be seen that the DCQPSO runs in attraction mode during the most of the iterations. Only when the diversity falls below the dlow do the particles fly in explosion mode. The explosion process is transitory, and once diversity is over the threshold, the population will return to attraction mode again.

\section{Experiment Results and Discussion}

We have tested the QPSO and DCQPSO on four widely known benchmark functions for testing the performance of different evolutionary optimization strategies. These functions are all minimization problems with minimum value zero. The four test functions are listed in Table 1. In all performance tests, the initial range of the population in all cases listed in Table 1 is asymmetry. Table 1 also lists $V_{\max }$ for original PSO.

The fitness value is set as function value and the neighborhood of a particle is the whole population. The population size is set to be 20 for all cases. We test original PSO with inertia weight (called Standard PSO or SPSO), QPSO and DCQPSOs for performance Comparison. We had 50 trial runs for every instance and recorded mean best fitness. The maximum number of iterations is set as 1000, 1500 and 2000 generations corresponding to the dimensions 10, 20 and 30 for first three functions, respectively, The dimension of the last functions is 2 and the maximum number of iterations is 2000 for this function. In performance test of the SPSO, the inertia weight 
Table 1. The table lists expression of benchmark function, the initial range of the population in the performance tests. The third column is the upbound of the velocity of the particle in the case of the SPSO.

\begin{tabular}{c|c|c|c|c}
\hline Functions & Formulations & Initial Range & $\mathbf{X}_{\max }$ & $\mathbf{V}_{\max }$ \\
\hline $\begin{array}{c}\text { Rosenbrock } \\
\text { function } f_{2}\end{array}$ & $f(x)_{2}=\sum_{i=1}^{n}\left(100\left(x_{i+1}-x_{i}^{2}\right)^{2}+\left(x_{i}-1\right)^{2}\right)$ & $(15,30)$ & 100 & 100 \\
\hline $\begin{array}{c}\text { Rastrigrin } \\
\text { function } f_{3}\end{array}$ & $f(x)_{3}=\sum_{i=1}^{n}\left(x_{i}{ }^{2}-10 \cos \left(2 \pi x_{i}\right)+10\right)$ & $(2.56,5.12)$ & 10 & 10 \\
\hline $\begin{array}{c}\text { Griewank } \\
\text { function } f_{4}\end{array}$ & $f(x)_{4}=\frac{1}{4000} \sum_{i=1}^{n}\left(x_{i}-100\right)^{2}-\prod_{i=1}^{n} \cos \left(\frac{\left(x_{i}-100\right)}{\sqrt{i}}\right)+1$ & $(300,600)$ & 600 & 600 \\
\hline $\begin{array}{c}\text { Shaffer's } \\
\text { function } f_{5}\end{array}$ & $f(\mathrm{x})_{7}=0.5+\frac{\left(\sin \sqrt{\mathrm{x}^{2}+\mathrm{y}^{2}}\right)^{2}-0.5}{\left(1.0+0.001\left(\mathrm{x}^{2}+\mathrm{y}^{2}\right)^{2}\right.}$ & $(30,100)$ & 100 & 100 \\
\hline
\end{tabular}

Table 2. Average best fitness and standard deviation of all algorithms on Rosenbrock function

\begin{tabular}{c|c|c|c|c}
\hline & Dimension & MAXITER & Mean Value & St. Dev. \\
\hline SPSO & & & & \\
& 10 & 1000 & 94.1276 & 194.3648 \\
& 20 & 1500 & 204.337 & 293.4544 \\
& 30 & 2000 & 313.734 & 547.2635 \\
QPSO & 10 & 1000 & 59.4764 & 153.0842 \\
& 20 & 1500 & 110.664 & 149.5483 \\
& 30 & 2000 & 147.609 & 210.3262 \\
DCQPSO1 & 10 & 1000 & 19.0109 & 29.5079 \\
& 20 & 1500 & 82.0134 & 84.4259 \\
& 30 & 2000 & 111.9926 & 164.3119 \\
DCQPSO2 & 10 & 1000 & 34.6391 & 63.4889 \\
& 20 & 1500 & 102.1606 & 178.6908 \\
& 30 & 2000 & 128.0084 & 160.3456 \\
\hline
\end{tabular}

Table 3. Average best fitness and standard deviation of all algorithms on Rastrigrin function

\begin{tabular}{c|c|c|c|c}
\hline & Dimension & MAXITER & Mean Value & St. Dev. \\
\hline \multirow{4}{*}{ SPSO } & 10 & & & \\
& & 1000 & 5.5382 & 3.0477 \\
& 20 & 1500 & 23.1544 & 10.4739 \\
QPSO & 30 & 2000 & 47.4168 & 17.1595 \\
& 10 & 1000 & 5.2543 & 2.8952 \\
& 20 & 1500 & 16.2673 & 5.9771 \\
DCQPSO1 & 30 & 2000 & 31.4576 & 7.6882 \\
& 10 & 1000 & 4.8308 & 2.4628 \\
& 20 & 1500 & 13.0424 & 4.8795 \\
DCQPSO2 & 30 & 2000 & 22.7104 & 5.5532 \\
& 10 & 1000 & 4.6831 & 3.6387 \\
& 20 & 1500 & 15.3056 & 11.8478 \\
& 30 & 2000 & 24.2655 & 6.4856 \\
\hline
\end{tabular}


$w$ is decreases linearly from 0.9 to 0.4 as in [17]. In performance tests for QPSO and DCQPSOs, the Contraction-Expansion Coefficient $\beta$ varies from 1.0 to 0.5 linearly when the algorithms are running. For DCQPSOs, this parameter control means that the population is in attraction mode. The threshold value of the diversity measure $d_{\text {low }}$ is to be 0.005. Moreover, in DCQPSO1, the value of $\beta_{0}$ is set as 2.0.

The mean values and standard deviations of best fitness for 50 runs of each function are recorded in Table 2 to Table 5. The numerical results show that the DCQPSOs works better on the first three functions than QSPO and SPSO. DCQPSO1 has slightly better performance than DCQPSO2. On Shaffer's function, the performances of DCQPSOs are not improved.

Table 4. Average best fitness and standard deviation of all algorithms on Greiwank function

\begin{tabular}{c|c|c|c|c}
\hline & Dimension & MAIXTER & Mean Value & St. Dev. \\
\hline \multirow{3}{*}{ SPSO } & & 1000 & & \\
& 10 & & 0.09217 & 0.0833 \\
& 20 & 1500 & 0.03002 & 0.03255 \\
QPSO & 30 & 2000 & 0.01811 & 0.02477 \\
& 10 & 1000 & 0.08331 & 0.06805 \\
& 20 & 1500 & 0.02033 & 0.02257 \\
DCQPSO1 & 30 & 2000 & 0.01119 & 0.01462 \\
& 10 & 1000 & 0.0781 & 0.0745 \\
& 20 & 1500 & 0.0189 & 0.0229 \\
DCQPSO2 & 30 & 2000 & 0.0090 & 0.0132 \\
& 10 & 1000 & 0.0655 & 0.0464 \\
& 20 & 1500 & 0.0202 & 0.0204 \\
& 30 & 2000 & 0.0087 & 0.0122 \\
\hline
\end{tabular}

Table 5. Average best fitness and standard deviation of all algorithms on Shaffer's function

\begin{tabular}{c|c|c|c|c}
\hline & & & Mean Value & \\
& Dimension & MAXITER & & St. Dev. \\
\hline SPSO & 2 & 2000 & $2.78 \mathrm{E}-04$ & 0.001284 \\
QPSO & 2 & 2000 & 0.001361 & 0.003405 \\
DCQPSO1 & 2 & 2000 & 0.0012 & 0.0032 \\
DCQPSO2 & 2 & 2000 & 0.0019 & 0.0039 \\
\hline
\end{tabular}

\section{Conclusion}

In this paper, we proposed a method of controlling the diversity measure of the QPSO. The methodology of DCQPSO is setting a threshold value for the diversity to prevent the particles from clustering. Controlling of the diversity has been testified to be a good technique for enhancing the performance of the QPSO. However, for other benchmark functions, such as Sphere function and Ackley function, the results of this method is poor, since the optima of these functions is easy to find and the local search 
ability is key to the performance of the algorithm. Annealing the diversity threshold d low could lead to improvements, because it may be an advantage to decrease dlow near the end of the optimization.

\section{References}

1. P. J. Angeline, "Evolutionary Optimization Versus Particle Swarm Optimization: Philosophy and performance Differences," Evolutionary Programming VII (1998), Lecture Notes in Computer Science 1447, pp. 601-610, Springer.

2. F. Van den Bergh, A. P. Engelbrecht, "A New Locally Convergent Particle Swarm Optimizer," 2002 IEEE International Conference on systems, Man and Cybernetics, 2002.

3. F. Van den Bergh, "An Analysis of Particle Swarm Optimizers," PhD Thesis. University of Pretoria, Nov 2001.

4. M. Clerc, "The Swarm and Queen: Towards a Deterministic and Adaptive Particle Swarm Optimization,” Proc. Congress on Evolutionary Computation 1999, pp. 1951-1957.

5. M. Clerc and J. Kennedy, "The Particle Swarm: Explosion, Stability, and Convergence in a Multi-dimensional Complex Space", IEEE Transaction on Evolutionary Computation, no. 6, pp. 58-73, 2002.

6. A. P. Engelbrecht and A. Ismail, "Training product unit neural networks, "Stability Control: Theory APPL., vol. 2, no. 1-2, pp.59-74

7. J. Kennedy, R. C. Eberhart, "Particle Swarm Optimization," Proc. IEEE Int'l Conference on Neural Networks, IV. Piscataway, NJ: IEEE Service Center, 1995, pp. 1942-1948.

8. J. Kennedy, "Sereotyping: Improving Particle Swarm Performance with cluster analysis," in Proc. 2000 Congress on Evolutionary Computation, pp. 1507-1512.

9. J. Kennedy, "Small worlds and Mega-minds: Effects of Neighborhood Topology on Particle Swarm Performance," Proc. Congress on Evolutionary Computation 1999, pp. 1931-1938.

10. P. N. Suganthan, "Particle Swarm Optimizer with Neighborhood Operator," Proc. 1999 Congress on Evolutionary Computation, pp. 1958-1962.

11. J. Sun et al, "Particle Swarm Optimization with Particles Having Quantum Behavior," Proc. 2004 Congress on Evolutionary Computation, pp. 325-331.

12. J. Sun et al, "A Global Search Strategy of Quantum-behaved Particle Swarm Optimization," Proc. 2004 IEEE Conference on Cybernetics and Intelligent Systems.

13. J. Sun et al, "Adaptive Parameter Control for Quantum-behaved Particle Swarm Optimization on Individual Level", Proceedings of 2005 IEEE International Conference on Systems, Man and Cybernetics, pp. 3049-3054.

14. R. K. Ursem: Diversity-Guided Evolutionary Algorithms, Proceedings of The Parallel Problem Solving from Nature Conference 2001.

15. J. Vesterstrom, J. Riget and T. Krink: Division of Labor in Particle Swarm Optimization. IEEE 2002 Proceedings of the Congress on Evolutionary Computation.

16. Y. Shi and R. Eberhart, "Empirical Study of Particle Swarm optimization," Proc. of Congress on Evolutionary Computation, 1999, 1945-1950.

17. Y. Shi, R. C. Eberhart, "A Modified Particle Swarm," Proc. 1998 IEEE International Conference on Evolutionary Computation, pp. 1945-1950. 